\title{
EVALUACIÓN DE LOS RESULTADOS OBTENIDOS DE ESTIMACIÓN DE RESERVAS DE MINERAL DE UN MODELO GEOESTADÍSTICO CON LOS SOFTWARE DATAMINE VERSUS MINESIGHT*
}

\author{
Cárdenas Paucarchuco, Julio; Mayor Pariona, Saúl² y Mayor Pariona, Jannete ${ }^{3}$ \\ Facultad de Ingeniería de Minas de la Universidad Nacional del Centro del Perú
}

\begin{abstract}
RESUMEN
El estudio consistió básicamente en analizar los resultados que se obtienen al realizar la estimación de reservas de minerales aplicando los software Datamine y Minesight, como muchos conocen los resultados que se obtienen a través de ellos podrían resultar relativamente diferentes, debido a que para la evaluación de reservas de mineral se pueden utilizar muchos criterios para el modelo geoestadístico y matemático. De ahí nació la necesidad de hacer un análisis de los resultados para determinar que software se acerca más a la realidad. El estudio consistió en lo siguiente: en primer lugar se creó una misma base de datos para los dos software en estudio; luego se desarrollaron los modelos geoestadísticos con cada uno, para luego estimar sus respectivas reservas, obteniéndose resultados diferentes. Al final se evaluaron los modelos matemáticos empleados y se comparó los resultados. Podemos concluir que los resultados obtenidos con los dos software no difieren significativamente por lo cual su utilización preferencial es indiferente, más bien la preferencia de uno u otro debe estar orientado a la disponibilidad del software y la presencia de personal capacitado adecuadamente para trabajar con ellos.
\end{abstract}

Palabras Clave: surpac, gemcom, geoestat, goesoft.

\section{EVALUATION OF THE OBTAINED RESULTS OF ESTIMATION OF MINERAL RESERVES OF A MODEL GEOESTADÍSTICO WITH SOFTWARES DATAMINE VERSUS MINESIGHT}

\begin{abstract}
This study basically consisted in analyzing the obtained results at the moment of collimating the mineral reserves applying data mine and mine sight software since many know the results that are obtained through them could be relatively different, because of in the evaluation of mineral reserves a lot of criterions can be used for the mothemotual and geostatistical models. From this very was born the analysis of the results to determine which software was closer to the reality. This study consisted of the following: first an only database was created for both software in study, then the geoslotistical models were developed with each one for a later study of their mineral reserves, getting different results and at the end, the mathematical models were evaluated and the results were compared we can conclude that the results obtained with the two software were not significantly different and therefore its preferential use is indifferent, but soother prefer one or the other should be directed to the availability of the software and the presence personal adequately trained to work with there.
\end{abstract}

Key words: surpac, gemcom, geoestat, goesoft.

* Este trabajo de investigación fue recibido el 20/04/2008 retornado para su revisión el 22/09/2008 y aprobado para su publicación el 27/02/2009.

1. E-mail: jcardenaspa@terra.com

2. E-mail: saulmayor@hotmail.com

3. E-mail: janettemp@hotmail.com 


\section{INTRODUCCIÓN}

El problema fundamental del estudio fue la existencia de muchos software de aplicación para la evaluación de reservas de minerales. Los resultados que ofrecen los mencionados programas de aplicación siempre resultan diferentes para el mismo modelo geoestadístico, es por esa razón que existe gran incertidumbre en las profesiones para tomar la decisión adecuada en la utilización de uno de estos programas.

Los resultados no son iguales debido a que cada programa o software aplica criterios diferentes y eso no quiere decir que uno de ellos estime mal las reservas, sino que en los modelos matemáticos se pueden combinar diversos criterios de acuerdo a las experiencias del profesional que hace uso de estos modelos, de ahí que los criterios son diferentes y los resultados también.

Debido a ésto, nace la necesidad de hacer un estudio de los resultados que ofrecen los softwares Datamine y Minesigth.

El problema general se puede plantear con la siguiente interrogante: ¿qué software se debe utilizar para la evaluación de reservas de mineral de un modelo geoestadístico, Datamine o Minesight?. La hipótesis planteada se formuló de la siguiente manera: para la estimación de reservas de mineral a partir de un modelo geoestadístico se debe utilizar el software Datamine, ya que ofrece resultados más confiables.

El impacto que se espera obtener como resultado de la presente investigación es reducir la incertidumbre en elegir un software determinado para la evaluación de reservas de mineral a partir de modelos geoestadísticos. Los usuarios directos potenciales de los resultados, serán todas las personas, profesionales de Ingeniería de Minas, Geología y Metalurgia; y empresas dedicadas actividad minera.

Por otra parte el objetivo establecido para el presente trabajo radica en definir que software se debe utilizar para la estimación de reservas a partir de un modelo geoestadístico, Datamine o Minesigth. Se utiliza el método científico: descriptivo, analítico, comparativo, experimental.

\section{MATERIAL Y MÉTODOS}

A continuación describimos el procedimiento incluyendo los métodos y materiales a seguir para la evaluación de los resultados obtenidos de estimación de reservas de mineral de un modelo geoestadístico con los software Datamine versus Minesight.

1. Elaboración de la base de datos de los taladros de exploración.

2. Modelamiento de los taladros con los software Datamine y Minesight.

3. Modelamiento del Yacimiento con los dos software en estudio.

4. Creación de los modelos geoestadístico en ambos software.

5. Estimación de reservas con los software Datamine y Minesight.

Evaluación de los resultados obtenidos de estimación de reservas de mineral de un modelo geoestadístico con los software Datamine versus Minesight.

\section{RESULTADOS}

\section{a. Elaboración de la base de datos de los taladros de exploración.}

\section{i) Base de datos de los taladros}

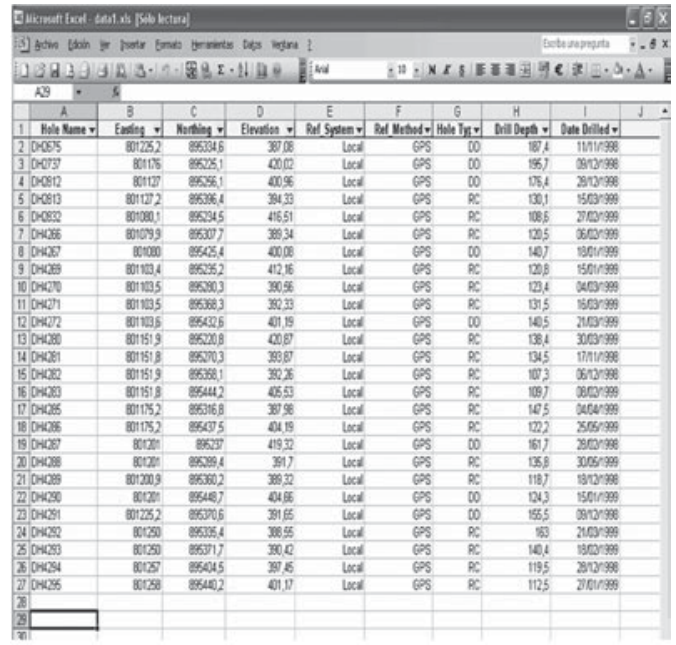

Figura 1. Base de datos del collar de los taladros (collars). 


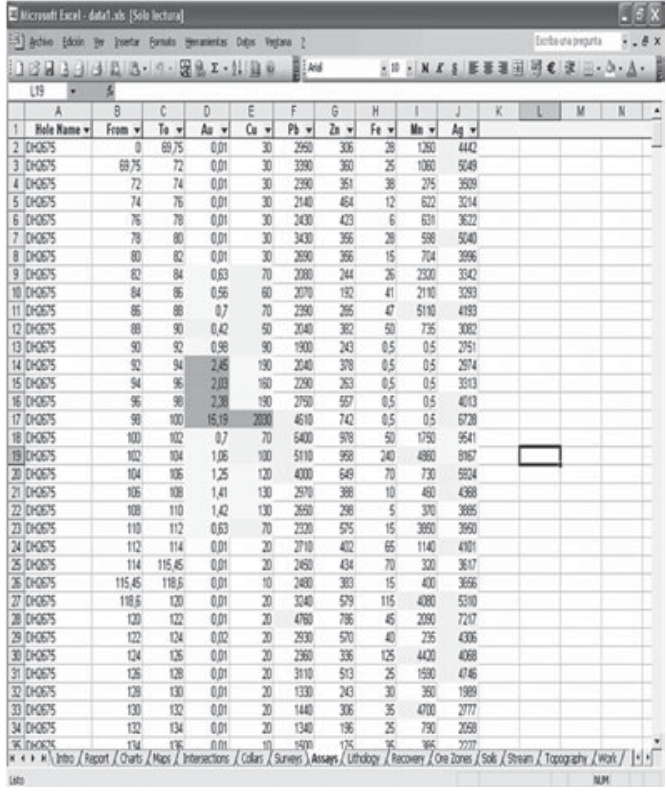

Figura 2. Base de datos de la dirección de los taladros (surveys).

\begin{tabular}{|c|c|c|c|c|c|c|c|c|}
\hline \multicolumn{8}{|c|}{ 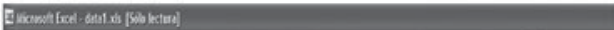 } & \multirow{2}{*}{$\frac{68 x}{.6 x}$} \\
\hline \multicolumn{4}{|c|}{ 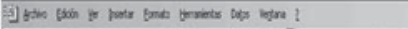 } & & & \multicolumn{2}{|c|}{ Eotausuppita } & \\
\hline \multicolumn{9}{|c|}{ 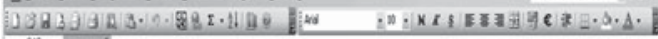 } \\
\hline \multicolumn{9}{|c|}{$612 \cdot \mathrm{s}$} \\
\hline$A$ & 8 & \begin{tabular}{l|l} 
& 0 \\
\end{tabular} & $E$ & $f$ & 6 & H & $T$ & $\mathrm{~T}$ \\
\hline \multicolumn{9}{|c|}{ 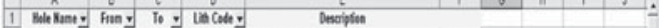 } \\
\hline 2 orss - & $\overrightarrow{5}$ & nj] Bast & \multicolumn{6}{|l|}{ conputery weed that } \\
\hline 30005 & ns & $\cos 30 \mathrm{x}$ & \\
\hline 4 otess & w3 & sant & \multicolumn{3}{|l|}{ 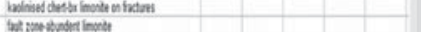 } & & & \\
\hline 5 onos & 8 & Font & \multicolumn{6}{|l|}{ 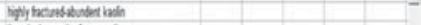 } \\
\hline 6 oross & $\infty$ & 100.1 Gousm & \multicolumn{6}{|l|}{ Inplatimentes coundet } \\
\hline 7 otes & 100,1 & 108,1 cksicht & \multicolumn{6}{|l|}{ bepitapgent } \\
\hline$B$ orest & 108,4 & 112 cksicte & \multicolumn{6}{|l|}{ Desprom } \\
\hline 9 oress & III & 10itheles & \multicolumn{6}{|l|}{ 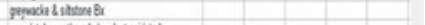 } \\
\hline Doters & 25 & $528 \mathrm{Bna}$ & \multicolumn{6}{|l|}{ 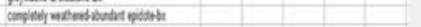 } \\
\hline 110003 & $\$ 2$ & W2ant & \multicolumn{6}{|l|}{ iclostadopy } \\
\hline 120603 & 22 & G Beat & \multicolumn{6}{|l|}{ 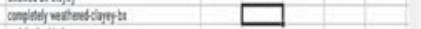 } \\
\hline B1300y & $\Leftrightarrow$ & sant & \multicolumn{6}{|l|}{ nodichous } \\
\hline 14 owey & क & 116 Coetr & \multicolumn{6}{|l|}{ 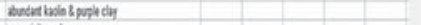 } \\
\hline 150603 & 116 & 1233 Siddet & \multirow{2}{*}{\multicolumn{6}{|c|}{ 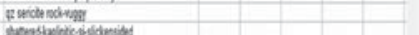 }} \\
\hline 150003 & {$[23$} & wogan: & 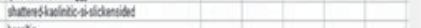 & & & & & \\
\hline 70052 & 0 & $15 \mathrm{Sal}$ & \multirow{2}{*}{\multicolumn{6}{|c|}{ batk }} \\
\hline Boren & 15 & 255Bant & viste img hadias & & & & & \\
\hline Bowen? & 25,5 & 33 Estate & 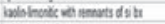 & & & & & \\
\hline Dowen & क3 & 05 Basat & pidela ing fotos & & & & & \\
\hline zores & 05 & $930 \mathrm{cot}$ & 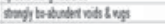 & & & & & \\
\hline zores? & 23 & Q1 Area & Ascintog biece & & & & & \\
\hline Bones? & \$:1 & G Coksict & 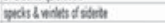 & & & & & \\
\hline a ONES & $\Leftrightarrow$ & Belstenth & porsons & & & & & \\
\hline X OESL & (2) & rockilckta & Stentrea & & & & & \\
\hline 8065 & $\pi$ & 617 Epten & 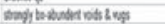 & & & & & \\
\hline 28 oren & 81) & $0 x$ Berca & ascinter toreces & & & & & \\
\hline xoles? & Q15 & III19 chet & 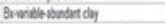 & & & & & \\
\hline$x$ oten & IIIs & 120100e & 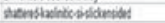 & & & & & \\
\hline$x$ orest & 0 & $28 \mathrm{od}$ & Inethed nok & & & & & \\
\hline 3100025 & $x i$ & xis one & at the theres ser end of itend & & & & & \\
\hline Dotest & $x$ & 726 aet & 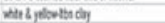 & & & & & \\
\hline mores & 725 & Q1 Buat & daptone gid dat & & & & & \\
\hline प्र ONes: & 8,1 & 6860 art & 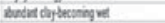 & & & & & \\
\hline 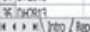 & $\log 2$ & $x / \sec$ & 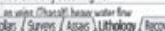 & & & & & to \\
\hline so & & & & & & & un & \\
\hline
\end{tabular}

Figura 3. Base de datos de del contenido metálico de los taladros (assays).

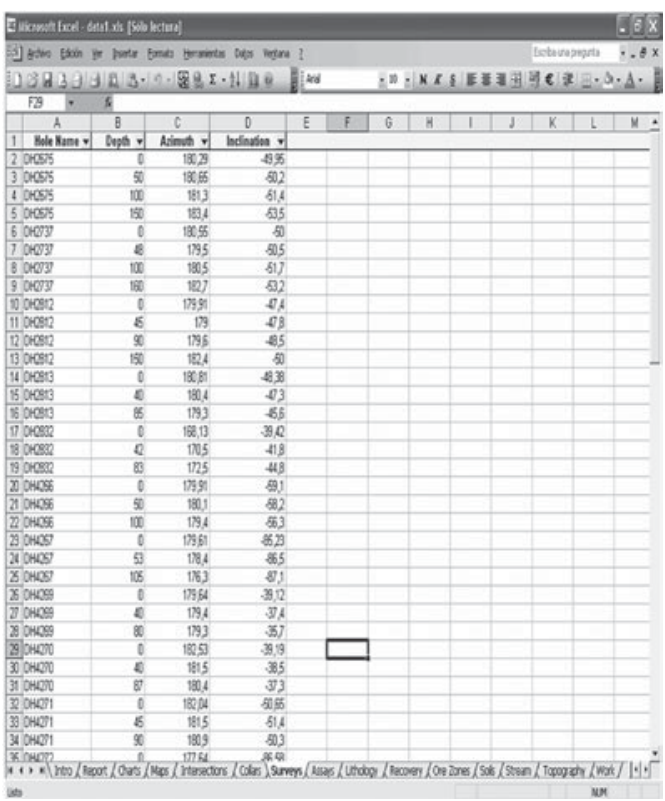

Figura 4. Base de datos del tipo de roca de los taladros (geology).

\section{ii) Otras bases de datos}

\begin{tabular}{|c|c|c|c|c|}
\hline 1 & $\mathrm{X}$ & $\mathbf{Y}$ & $\mathbf{Z}$ & \\
\hline 2 & 800256,3368 & 895004,1737 & 375 & \\
\hline 3 & 800260,8651 & 895000,6628 & 375 & \\
\hline 4 & 800278,7547 & 894991,9214 & 375 & \\
\hline 5 & 800282,109 & 894990,8618 & 375 & \\
\hline 6 & 800296,6444 & 894986,4912 & 375 & \\
\hline 7 & 800314,534 & 894982,3854 & 375 & \\
\hline 8 & 800332,4237 & 894981,1934 & 375 & \\
\hline 9 & 800350,3133 & 894978,0147 & 375 & \\
\hline 10 & 800368,203 & 894975,7631 & 375 & \\
\hline 11 & 800368,203 & 894975,8956 & 375 & \\
\hline 12 & 800350,3133 & 894987,8156 & 375 & \\
\hline 13 & 800346,1204 & 894990,8618 & 375 & \\
\hline 14 & 800332,4237 & 894997,4841 & 375 & \\
\hline 15 & 800316,91 & 895007.8148 & 375 & \\
\hline 16 & 800314,534 & 895009,2717 & 375 & \\
\hline 17 & 800296,6444 & 895021,854 & 375 & \\
\hline 18 & 800293,7094 & 895024,7677 & 375 & \\
\hline 19 & 800278,7547 & 895036,158 & 375 & \\
\hline 20 & 800271,2075 & 895041,7207 & 375 & \\
\hline 21 & 800260,8651 & 895046,3563 & 375 & \\
\hline 22 & 800254,8077 & 895044,4245 & 375 & \\
\hline 23 & 800260,84 & 894929,5473 & 380 & \\
\hline 24 & 800260,8651 & 894929,5398 & 380 & \\
\hline 25 & 800278,7547 & 894926,2287 & 380 & \\
\hline 26 & 800296,6444 & 894924,3745 & 380 & \\
\hline 27 & 800314,534 & 894923.5798 & 380 & \\
\hline 28 & 800332,4237 & 894923,4474 & 380 & \\
\hline 29 & 800350,3133 & 894923,9771 & 380 & \\
\hline 30 & 800368,203 & 894925,3016 & 380 & \\
\hline 31 & 800386,0926 & 894927,2883 & 380 & \\
\hline 32 & 800403,9823 & 894930,2021 & 380 & \\
\hline 33 & 800421,8719 & 894934,1754 & 380 & \\
\hline 34 & 800439,7616 & 894939,3408 & 380 & \\
\hline 35 & 8nn441 299 & $894947 \cap n 3$ & $38 n$ & \\
\hline
\end{tabular}




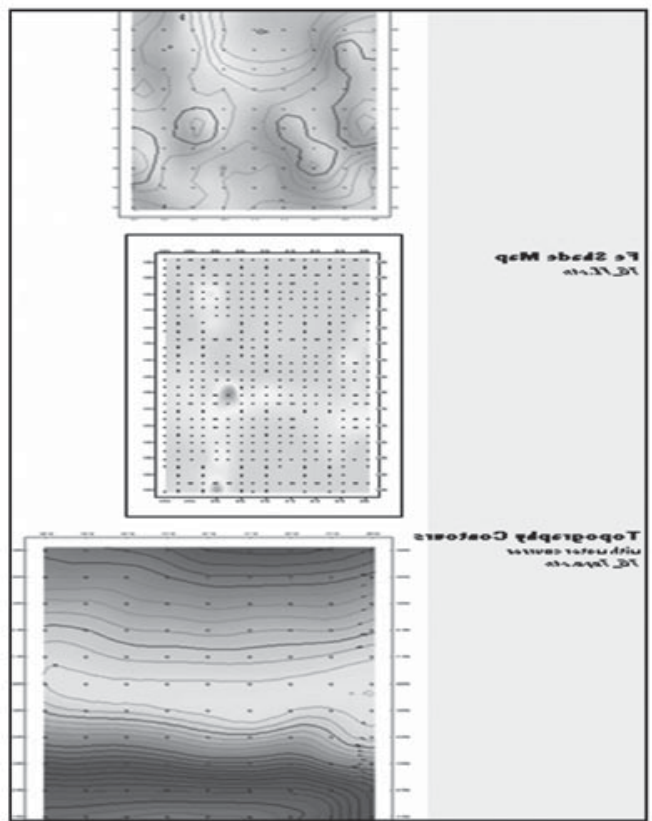

Figura 5 Base de datos de la topografía del terreno.

\section{b. Estimación de reservas con el software Datamine Studio}

i) Modelamiento digital del proyecto

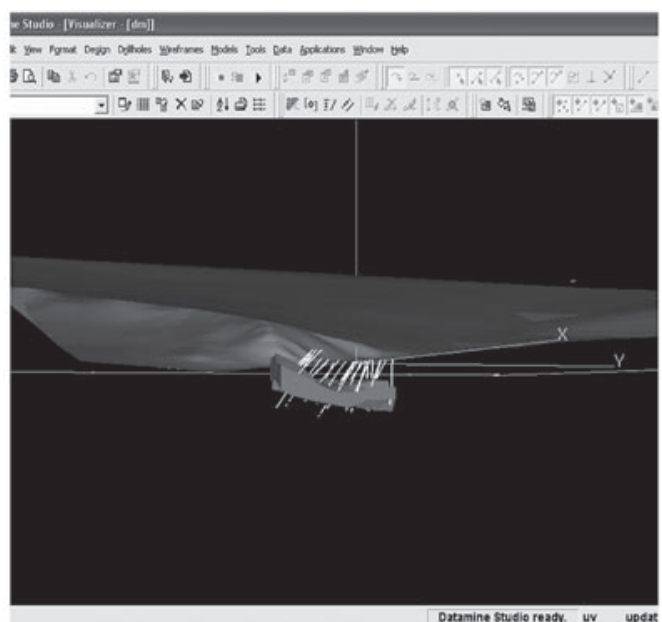

Figura 6. Modelamiento de taladros, zona topográfica y yacimiento. ii) Estimación de reservas por el método geoestadístico

Tabla 1. Parámetros utilizados en el modelamiento geoestadístico (dimensiones del proyecto).

\begin{tabular}{llll}
\hline & \multicolumn{1}{c}{$\mathbf{X}$} & \multicolumn{1}{c}{$\mathbf{~}$} & \multicolumn{1}{c}{$\mathbf{Z}$} \\
\hline Pto. mínimo & 800254.8125 & 894458.5000 & 1.0000 \\
Pto. Máximo & 802526.7500 & 896108.6875 & 510.0000 \\
Rango & 2271.9375 & 1650.1875 & 510.0000 \\
Tamaño de & 10 & 10 & 10 \\
celdas & & & \\
Nro. de celdas & 228 & 165 & 51 \\
\hline
\end{tabular}

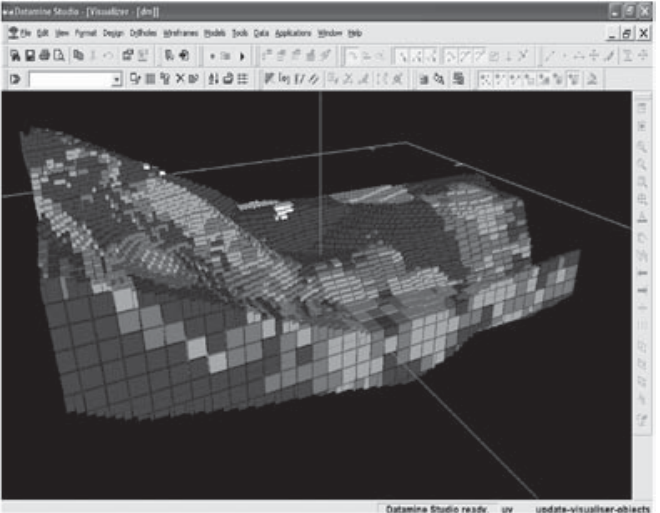

Figura 7. Modelo de bloks.

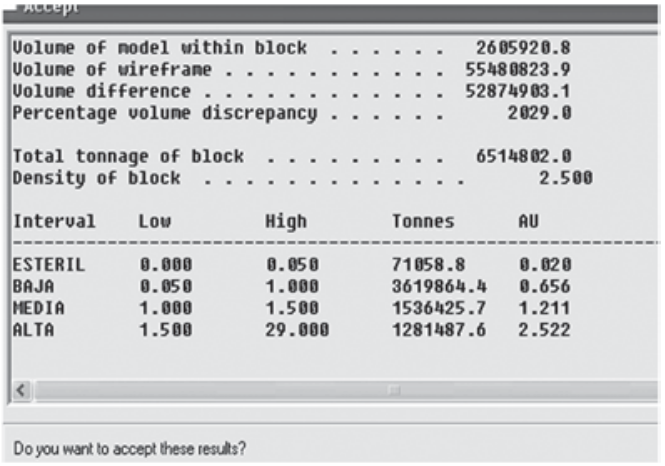

Figura 8. Resultados obtenidos con Datamine.

\begin{tabular}{|c|c|c|c|c|c|c|}
\hline Recoord & BLOK DENSTY & VOLUMETONNES & NTERVALO & CATEGORY & LOWER & UPPER \\
\hline 1 & \begin{tabular}{l|l}
1 & 25 \\
\end{tabular} & \begin{tabular}{l|l|} 
& 71400,450018 \\
\end{tabular} & 1 & Estenl & & 0,5 \\
\hline 2 & 125 & \begin{tabular}{ll|l|}
3619000,67 & 0,33 \\
\end{tabular} & 2 & Baja & 0,5 & 1 \\
\hline 4 & 125 & \begin{tabular}{ll|l|}
1542448,45 & 1,18 \\
\end{tabular} & 3 & Media & & 15 \\
\hline 4 & 125 & $\begin{array}{lll}128163356 & 2,48 \\
\end{array}$ & 4 & Alla & 15 & 29 \\
\hline
\end{tabular}

Figura 9. Resultados obtenidos con Minesight 


\section{DISCUSIÓN}

La diferencia en los resultados obtenidos (Datamine - Minesight) podemos establecerlo de la siguiente manera:

- Para la categoría estéril:

$71058,8-71400,45=-341,65$ toneladas, con un contenido de $0,020-0,018=0,002$ gramos por tonelada de oro.

- Para la categoría baja:

$3619864,4-3619800,67=63,73$ toneladas, con un contenido de $0,656-0,63=0,026$ gramos por tonelada de oro.

- Para la categoría media:

$1536425,7-1542448,45=-6022,75$ toneladas, con un contenido de 1,211 - 1,18 = 0,031 gramos por tonelada de oro.

- Para la categoría alta:

$1281487,6-1281633,56=-145,96$ toneladas, con un contenido de 2,522 - 2,48 =0,042 gramos por tonelada de oro.

La diferencia encontrada referida a la estimación de reservas por toneladas es mínima en todas las categorías, por otro lado existe una ligera variación con respecto al contenido metálico para las categorías baja, media y alta; mas no en la categoría estéril, esta variación también se puede asumir que es indiferente si hacemos el análisis desde el punto de vista de la construcción de los modelos geoestadísticos que sirvieron para la evaluación de reservas, como se utilizaron diferentes modelos geoestadísticos elaborados en cada software estos modelos de ninguna manera podrán ser los mismos, ya que es imposible tomar puntos iguales para cada software, es por esa razón que siempre habrá cierta variación en cuanto a toneladas y contenido metálico.

\section{CONCLUSIONES}

- Los resultados obtenidos con el software Datamine fueron: para la categoría estéril 71058,8 toneladas con un contenido de 0,020 gramos por tonelada de oro, para la categoría baja 3619864,4 toneladas con un contenido de 0,656 gramos por tonelada de oro, para la categoría media 1536425,7 toneladas con un contenido de 1,2111 gramos por tonelada de oro y para la categoría alta 1281487,6 toneladas con un contenido de 2,522 gramos por tonelada de oro.

- Los resultados obtenidos con el software Minesight fueron: para la categoría estéril 71400,45 toneladas con un contenido de 0,018 gramos por tonelada de oro, para la categoría baja 3619800,67 toneladas con un contenido de 0,63 gramos por tonelada de oro, para la categoría media 1542448,45 toneladas con un contenido de 1,18 gramos por tonelada de oro y para la categoría alta 1281633,56 toneladas con un contenido de 2,48 gramos por tonelada de oro.

- La diferencia de los resultados obtenidos con los software Minesight y Datamine fueron: para la categoría estéril 341,65 toneladas con una diferencia de contenido de 0,002 gramos por tonelada de oro, para la categoría baja 63,73 toneladas con una diferencia de contenido de 0,026 gramos por tonelada de oro, para la categoría media 6022,75 toneladas con una diferencia de contenido de 0,031 gramos por tonelada de oro y para la categoría alta145,96 toneladas con una diferencia de contenido de 0,042 gramos por tonelada de oro.

- Podemos concluir que los resultados obtenidos con los dos software no difieren significativamente, y están dentro de los márgenes aceptables de error que para estos casos se considera el $1 \%$ de las toneladas cubicadas, por lo cual la utilización preferencial de uno de los software es indiferente, más bien la preferencia de uno u otro software debe estar orientado a la disponibilidad del software y la presencia de personal capacitado adecuadamente para trabajar con los software.

\section{LITERATURA CITADA}

Bleines, C; Deraisme, J; Geffroy, F; Parseval, S; Rambert F., Renard D. y Touffait, Y. 2001. ISATIS Software Manual. Geovariance and Ecole des Mines de Paris. Paris, January, 2001, 531 pp. 
Chen, Y. and Xiguo J. 2001. Semivariogram fitting with linear programming. Computers \& Geosciences, Volume 27, Issue 1, February 2001, Pages 7176.

Clark, I. 1977. Practical Geostatistics. Geostokos Limited, United Kingdom. Versión digital descargada. http://uk.geocities.com/drisobelclark/PG1979. $119 \mathrm{pp}$.
David, M. 1977. Geostatistical ore reserve estimation. Elsevier Scientific Publishing Company. New York, 1977. 364 pp.

www.datamine.co.uk

www.gemcom.com

www.surpac.com 